\title{
Novel fullerene platinum alkynyl complexes with high second-order nonlinear optical properties as a springboard for NLO-active polymer films
}

Claudia Dragonetti, ${ }^{\dagger, \ddagger}$ Alessia Colombo, ${ }^{\dagger *}$ Mattia Fontani, ${ }^{\dagger}$ Daniele Marinotto, ${ }^{\ddagger}$ Filippo Nisic, ${ }^{\dagger}$ Stefania Righetto, ${ }^{\dagger}$ Dominique Roberto, ${ }^{\dagger, \dagger}$ Francesco Tintori ${ }^{\dagger}$ and Simona Fantacci ${ }^{¥}$

† Dipartimento di Chimica and Centro SmartMatLab dell’Università degli Studi di Milano, UdR INSTM di Milano, ${ }^{\dagger}$ ISTM-CNR, via Golgi 19, I-20133 Milano, Italy. ${ }^{¥}$ Computational Laboratory for Hybrid/Organic Photovoltaics (CLHYO), CNR-ISTM, Via Elce di Sotto 8, I-06213, Perugia, Italy. 
Table S1 Energy (eV) of the first HOMOs and LUMOs and the HOMO-LUMO of compounds 1-7

\begin{tabular}{|l|c|c|c|c|c|c|c|}
\hline & $\mathbf{1}$ & $\mathbf{2}$ & $\mathbf{3}$ & $\mathbf{4}$ & $\mathbf{5}$ & $\mathbf{6}$ & $\mathbf{7}$ \\
\hline LUMO+1 & -3.38 & -3.38 & -3.36 & -3.36 & -3.36 & -3.38 & -3.37 \\
\hline LUMO & -3.44 & -3.43 & -3.42 & -3.42 & -3.42 & -3.43 & -3.43 \\
\hline HOMO & -5.97 & -5.97 & -5.44 & -5.56 & -5.14 & -5.59 & -5.18 \\
\hline HOMO-n & -6.12 & -6.12 & -5.92 & -5.94 & -5.50 & -5.98 & -5.82 \\
& $\mathrm{n}=2$ & $\mathrm{n}=2$ & $\mathrm{n}=4-5$ & $\mathrm{n}=4-5$ & $\mathrm{n}=4-5$ & $\mathrm{n}=2-3$ & $\mathrm{n}=4-5$ \\
& -6.13 & -6.13 & -6.11 & -6.11 & -6.11 & $-6.12 /-$ & -6.12 \\
\hline $\mathbf{E}_{\text {HOMO-LUMO }}$ & 2.53 & 2.54 & 2.02 & 2.14 & 1.72 & 2.16 & 1.75 \\
\hline
\end{tabular}

